\title{
THE GENETIC AND MOLECULAR STUDIES OF HEPATITISC VIRUS: A REVIEW
}

\author{
*1 Rogo, L.D., ${ }^{1}$ Akogwu, S., ${ }^{2}$ Umar, U. Z., ${ }^{3}$ Aliyu, A.M. and ${ }^{4}$ Aminu, B. M. \\ ${ }^{1}$ Department of Haematology and Blood Group Serology, School of Medical Laboratory Sciences, \\ Ahmadu Bello University Teaching Hospital, Zaria. \\ ${ }^{2}$ Department of Biology, Sa'adatu Rimi College of Education, Kano. \\ ${ }^{3}$ Department of Applied Sciences, College of Science \& Technology, P.M.B.2021 Kaduna Polytechnic. \\ ${ }^{4}$ Department of Biology, Kano State University of Science and Technology, Wudil \\ *Correspondence author: lawaldahirurogo@yahoo.com
}

\begin{abstract}
The role of Hepatitis viruses, particularly Hepatitis $c$ virus (HCV) as human pathogen and their transmission have been of interest over the years. The virus is a small (55-65nm in size), included in Group IV, enveloped, positive sense, single stranded RNA virus, the family Flaviviridae, genus Hepacivirus, and Hepatitis $c$ virus type species. Based on genetic differences between HCV isolates, the virus species is classified into six genotypes (1-6) with several subtypes within each genotype (represented by letters). Persistent infection with Hepatitis $c$ virus (HCV) has emerged as one of the primary causes of chronic liver disease with an estimated 170 million people infected by HCV, more than 4 times the number of people living with HIV throughout the world. The present review looks at the genetic and molecular nature of this virus with the view to provide more information about its biology which may be useful to the present and feature researchers.
\end{abstract}

Key words: Hepatitis c virus, biology, genome, chronic, liver, disease

\section{INTRODUCTION}

Despite the rapid scientific progress in understanding the biology of viral illness, viral liver disease remains a common and challenging problem for physicians and their patients (Alter and Seeff, 2000). Persistent infection with Hepatitis c virus (HCV) has emerged as one of the primary causes of chronic liver disease with an estimated 170 million people infected by HCV, more than 4 times the number of people living with HIV throughout the world $(\mathrm{WHO}, 2000)$. Of the typical hepatitis viruses, chronic infection with Hepatitis c virus remains one of the most important clinical and public health problems (El-Zayadi et al., 2004). In the western world, chronic damage from Hepatitis $\mathrm{c}$ is the primary cause of the end stage liver disease requiring liver transplantation (Niederan et al., 1998). The discovery of HCV in 1989 was a major breakthrough. Before that point, it was clear that a major cause of acute hepatitis after a blood transfusion was neither related to Hepatitis A nor to Hepatitis B- hence the early names for this disease, non- $A$, non-B hepatitis (Simmonds et al., 2005).

In the mid 1970's Harvey J. Alter and his research team demonstrated that most post-transfusion hepatitis cases were not due to hepatitis A or B viruses. Despite this discovery, international research efforts to identify the virus, initially called non-A, non$B$ hepatitis (NANBH), failed for the next decade. In 1987 Michael Houghton, Qui-Lim Choo, and George Kuo at Chiron Corporation, collaboration with Dr. D.W. Bradley from CDC, utilised a novel molecular cloning approach to identify the unknown organism
(Sharma,2010). In 1988, the virus was confirmed by Alter by verifying its presence in a panel of NANAH specimens. In April of 1989, the discovery of the virus, re-named Hepatitis C virus (HCV), (Choo et al., 1989; Kuo et al., 1989). The virus belongs to the family Flaviviridae and Hepacivirus genus (Jawezt et al., 2004).

\section{Virology of Hepatitis C Virus Virion}

Hepatitis $\mathrm{C}$ virus ( $\mathrm{HCV}$ ) is a member of the Hepacivirus genus, of the Flaviviridae family (Meir and Ramadori, 2009). It is a small (55-65nm in size), enveloped, positive sense polarity, single stranded RNA virus. The viral particle consist of a core of genetic material (RNA), surrounded by an icosahedral protective shell of protein, and further encased in a lipid (fatty) envelope of cellular origin. Two viral envelope glycoproteins, E1 and E2, are embedded in the lipid envelope (Op De Beeck and Dubuisson, 2003).

\section{Genome Organisation of HCV}

Hepatitis $\mathrm{C}$ virus has a positive sense RNA that consists of a single open reading frame of 9600 nucleoside bases (Kato, 2000). At the $5^{\prime}$ and $3^{\prime}$ ends of the RNA are the UTR regions that are not translated into proteins but are important to translation and replication of the viral RNA. The 5' UTR has a ribosome binding site (Jubin, 2001), (IRESInternational Ribosome Entery Site) that starts the translation of a 3000 amino acid containing protein that is later cut by and viral proteases into active structural and non-structural smaller protein (Jawezt et al., 2004; Dubuisson, 2007). 


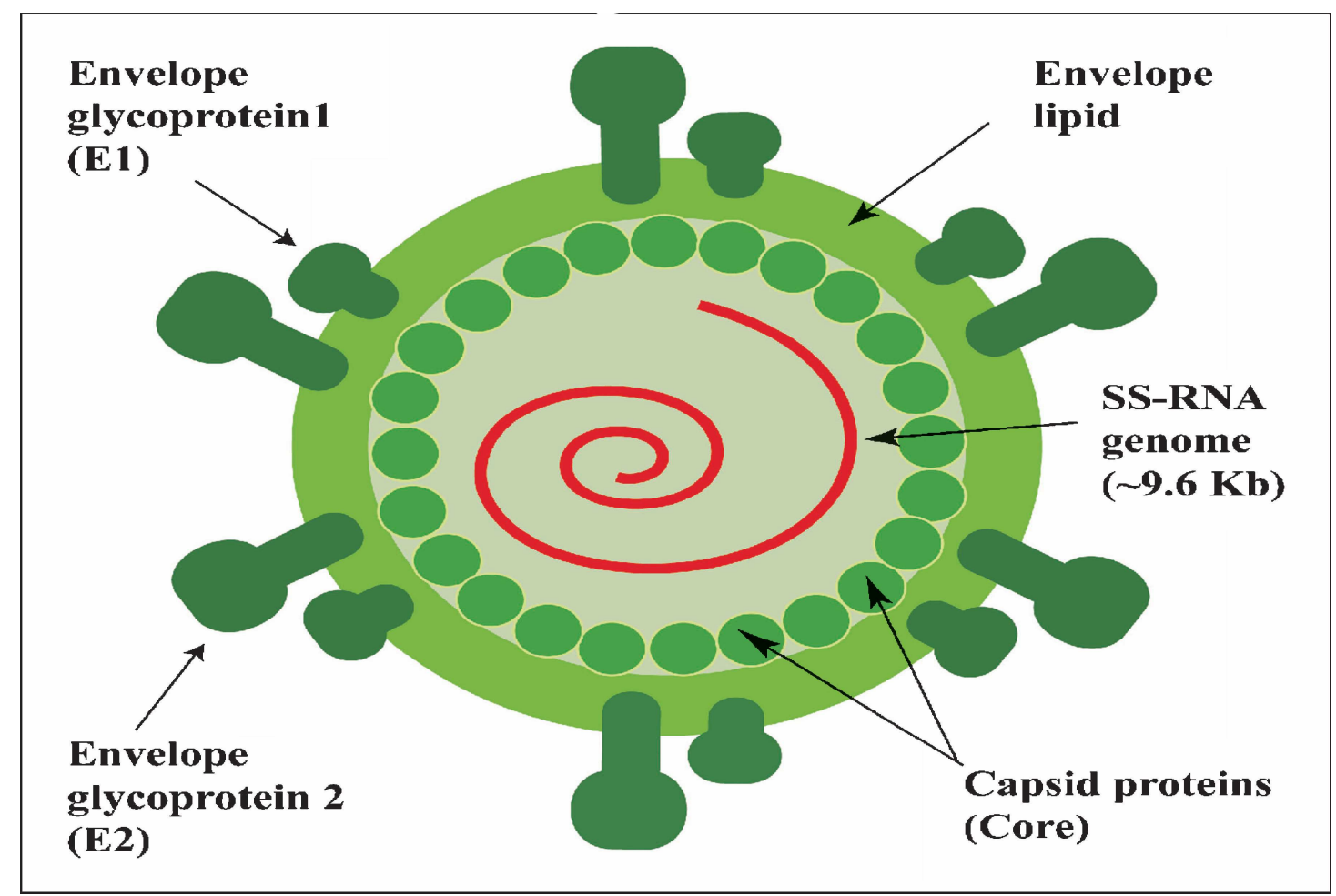

Figure 1. Hepatitis C virus particle structure: The HCV core protein interacts with viral genomic RNA to form the nucleocapsid. Two membrane-associated envelope glycoproteins, E1 and E2 are embedded in a lipid envelope which is derived from the host.

The 5'- and the 3'-NTRs of the genome are highly conserved and contain control elements for translation of the viral polyprotein and replication. The $5^{\prime}$ UTR $(+)$ is $~ 341$ nucleotides in length and contains an internal ribosomal entry site (IRES). The HCV IRES is folded into four stem-loop motifs which are called as I, II, III and IV. The IRES is required for capindependent translation of viral RNA, which is carried out by host cell ribosome. The domain IIId of the IRES constitutes the key anchoring site for the $40 \mathrm{~S}$ subunit (Lukavsky et al., 2000). The IRES domains IIIIV have also been shown to be an activator of protein kinase R (PKR) (Shimoike et al., 2009). However, this activation does not interfere with cap-independent translation of HCV viral proteins. HCV core protein was reported to interact with the $5^{\prime}$-NTR of plus-strand RNA (Fan et al., 1999). However, recent work with JHF1 viral RNA suggested that its 5'-NTR (+) does not contain RNA packaging signals (Friebe and Bartenschlager, 2009) and other authors further speculate that it may reside in the RNA region encoding the replicase. The $3^{\prime}-$ UTR $(+)$ is around 200nt and is involved in RNA replication. Three different domains can be recognized in this U TR: (I) a poly (U/UC) tract with an average length of 80 nucleotides (nt), (ii) a variable region, and (iii) a virtually invariant 98-nt X-tail region made up of 3 stem-loops (3'SLI, 3'SLII and 3'SLIII). The 3'-UTR can robustly stimulate IRES dependent translation in human hepatoma cell lines (Song et al., 2006). Recent studies have recognized that various stemloop structures exist in the negative strand $3^{\prime}$-NTR. This region is recognized by the viral polymerase as the initiation site for plus-strand synthesis of the HCV genome (Ye et al., 2005). A recent study identified a cellular factor called Far-upstream element (FUSE) binding protein (FBP) which binds to $3^{\prime}$ NTR by interacting with the poly (U) tract (Zhang et al., 2008). The importance of long-range RNARNA interactions in the modulation of HCV lifecycle has been well documented. Within the 3 '-end of the nonstructural protein 5B (NS5B) coding sequence, a cisacting replication element (CRE) was discovered (You et al., 2004). This CRE is called as SL9266 (or 5BSL3.2) and its disruption blocks RNA replication (Friebe et al., 2005). Mutual long range binding with both $5^{\prime}$ and $3^{\prime}$ sequences is suggested to stabilize the CRE at the core of a complex pseudoknot (Deviney et al., 2008). Non coding RNA molecules or microRNAs (miR) are important in the control of gene expression and regulation. MicroRNA, miR-122 is specifically expressed and is found to be abundant in the human liver (Jopling, 2008). A recent discovery showed binding of a miRNA (miR-122) to the 5'-UTR of HCV. Sequestration of miR-122 in liver cell lines strongly reduced HCV translation, whereas its addition stimulated translation via direct interaction of miR-122 with two sites in the 5'-UTR (Kruger et al., 2001). These studies have generated a lot of interest in the role of miR-122 in HCV multiplication and its potential as a therapeutic target. A role for proteasome alphasubunit PSMA7 in regulating HCV IRES mediated translation has also been demonstrated (Kruger et al., 2001). These host factors require further scrutiny to be considered as candidates for drug targets. 
The single open reading frame is expressed $\mathrm{s}$ a polyprotein that gets processed; the positions of structural and non-structural domains. HVR-1 represents the highly variable region of an envelope

\section{REFERENCES}

Alter,H. J. and Seeff, L.B. (2000). Persistence and sequele in Hepatitis $\mathrm{C}$ virus infection: a perspective on the long term outcome. Liver Disease 20:17-25.

Choo Q, Kuo G, Weiner A, Overby L, Bradley D, Houghton $M$ (1989). "Isolation of a CDNA clone derived from a blood-borne non-A, non-B viral hepatitis genome." Science 244 (4902): 359-362.

Deviney, S., Tuplin, A., Struthers, M., Armstrong, V., Elliott, R. M. and Simmonds, P. (2008). A hepatitis $C$ virus cis-acting replication element forms a long-range RNA-RNA interaction with upstream rna sequences in ns5b. Journal of Virology 82: 9008-9022.

Dubuisson, J. (2007). Hepatitis C virus proteins. World Journral of Gastroenterology 13(17): 24062415.

El-Zayadi, A., Osaima, S., Hamdy, H., El-Tawil, A., Hanaa, M. B., Attia, M. and Saeed, A. (2004). Impact of cigarette smoking on response to interferon therapy in chronic Hepatitis C Egyptian patients. World Journal of GastrOenterology 10(20):29632966.

Fan, Z., Yang, Q. R., Twu, J. S., Sherker, A. H. (1999). Specific in vitro association between the hepatitis C viral genome and core protein. Journal of Medical Virology 59: 131-134.

Friebe, P., Boudet, J., Simorre, J. P. and Bartenschlager, R. (2005). Kissingloop interaction in the 3 ' end of the hepatitis $C$ virus genome essential for RNA replication. Journal of Virology $79: 380-$ 92.

Friebe P, Bartenschlager, R. (2009). Role of rna structures in genome terminal sequences of the hepatitis $\mathrm{C}$ virus for replication and assembly. Journal of Virology 83: 11989-1195.

Jawetz, Melnick, and Adelberg's (2004). Medical Microbiology. In: Geo, F. B., Karen, C. C., Janet, S. B. and Stephen, A. M. $23^{\text {rd }}$ Int. edition Pp. 466-486 McGraw Hill publisher.

Jopling, C.L. (2008). Regulation of hepatitis C virus by microrna-122. Biochemistry Society Trans 36: 1220-1223.

Jubin, R. (2001). Hepatitis C IRES: translating translation into a therapeutic target. Current Opinion on Molecular Theraphy 3(3): 278-287.

Kato, N. (2000). Genome of human Hepatitis c virus (HCV): gene organisation, sequence diversity, and variation. Microbial Comparative Genomics 5(3):129-151.

Kruger, M., Beger, C., Welch, P. J., Barber, J. R., Manns, M. P. and Wong- Staal, F. (2001). Involvement of proteasome alpha-subunit psma7 in hepatitis $C$ virus internal ribosome entry site-mediated translation. Molecular Cell Biology 21 : 83578364.

Kuo G, Choo Q, Alter H, Gitnick G, Redeker A, Purcell R, Miyamura T, Dienstag J, Alter $M$, Stevens C (1989). "An assay for circulating antibodies to a glycoprotein. The genome organisation as shown in figure 2 below is $5^{\text {IUTR }}$ C-E1-E2-NS2-NS3-NS4A-NS4BNS5A-NS5B-3'URT (Jawezt et al., 2004; Lindenbach and Rice, 2005).

major etiologic virus of human non- $A$, non-B hepatitis.". Science 244 (4902): 362-4.

Lindenbach, B. and Rice, C. (2005). Unravelling hepatitis $C$ virus replication from genome to function. Nature 436 (7053): 933-938.

Lukavsky, P. J., Otto, G. A., Lancaster, A. M., Sarnow. P, and Puglisi, J. D. (2000) Structures of two RNA domains essential for hepatitis $C$ virusinternal ribosome entry site function. Nature Structure Biology 7: 1105-1110.

Meier, V. and Ramadori, G. (2009). Hepatitis C virus virology and new treatment targets. Expert Review on Antiviral Infection Therapy 7 (3):329-350.

Niederan,C., Lane, S. and Heitges, T. (1998). Prognosis of chronic Hepatitis C: results of a large, prospective cohort study. Hepatology 28:16871695.

Op De Beeck, J. and Dubuisson, J.(2003). Topology of hepatitis $C$ virus envelope glycoproteins. Review Medical Virology 13(4): 233-241.

Sharma, S. D. (2010). Hepatitis C virus: Molecular biology and current therapeutic options. Indian Journaal of Medical Research 131: 17-34.

Shimoike, T., McKenna, S. A., Lindhout, D.A. and Puglisi, J.D. (2009). Translational insensitivity to potent activation of pkr by HCV IRES RNA. Antiviral Res.

Simmonds, P., Bukh, J., Combet, C., Deleage, G., Enomoto, N., Feistone, S., Halfon, P., Inchauspe, G., Kuiken, C., Thiel, H., Viazov, S., Weiner, A. and Widell, N. (2005). Consensus proposal for a unified system of nomenclature of Hepatitis C virus genotypes. Hepatology 42(4):962-973.

Song, Y., Friebe, P., Tzima, E., Junemann, C., Bartenschlager, R, and Niepmann, M. (2006). The hepatitis C virus RNA 3'-untranslated Sharma: New therape utic approaches for HCV 29 region strongly enhances translation directed by the internal ribosome entry site. Journal of Virology 80: 11579-11588.

World Health Organisation (2000). Hepatitis C - global prevalence (update). Weekly Epidemiological Record 75:18-19.

Ye, L., Timani, K. A., Kong, L., Yang, X., Liao., Q, and $\mathrm{Wu}$, J. (2005). Two cisacting elements in negative RNA strand of hepatitis $C$ virus involved in synthesis of positive RNA strand in vitro. Acta Virology 49 : 83-90.

You, S., Stump, D. D., Branch, A. D. and Rice, C. M. (2004). A cis-acting replication element in the sequence encoding the ns5b RNAdependent RNA polymerase is required for hepatitis $C$ virus RNA replication. Journal of Virology 78 : 135266.

Zhang, Z., Harris, D. and Pandey, V. N. (2008). The fuse binding protein is a cellular factor required for efficient replication of hepatitis $C$ virus. Journal of Virology 82: 5761-5773. 